\title{
Whites Science
}

\author{
A Scientific Erudition
}

\section{EFFECTORANNOTATOR, A SCRIPT FOR AN EASY AND AUTOMATED IDENTIFICATION AND COMPARISON OF THE TYPE III SECRETION SYSTEM EFFECTORS OF PSEUDOMONAS SYRINGAE}

\author{
Simone Marcelletti ${ }^{1 *}$, Marco Scortichini ${ }^{1,2}$, Giuseppe Firrao ${ }^{3}$ \\ ${ }^{1}$ C.R.E.A.- Research Centre for Fruit Tree, Via di Fioranello, 52, 00134 Rome, Italy \\ ${ }^{2}$ C.R.E.A.- Research Centre for Fruit Tree, Via Torrino, 3, 81100 Caserta, Italy \\ ${ }^{3}$ Department of Agricultural Sciences and Environment, University of Udine, Via delle Scienze, 208, 33100, \\ Udine, Italy
}

\begin{abstract}
The type III secretion system of Pseudomonas syringae is a biological machinery that plays a fundamental role in causing diseases to both wild and cultivated plants through the releasing of effector proteins into the plant cell. Problems with the correct identification and comparison of effectors are usually encountered when a putative effector is annotated, particularly during the automated annotation in genome sequencing project. To improve the reliable identification of effector proteins and the address of their identity by homology comparison with the type III secretion system effectors of Pseudomonas syringae, we have developed and set up a program that enables fast and automated protein effectors search and comparison. EffectorAnnotator is free and open source software, available under a GPLv2 licence. EffectorAnnotator can be downloaded and installed from http://www.atlasplantpathogenicbacteria.it/ written in Perl and tested on Linux.
\end{abstract}

Keywords: Genomic annotation; Pseudomonas syringae; Effectors; Bioinformatics

\section{INTRODUCTION}

Pseudomonas syringae is a polyphagous bacterial phytopathogen able to infect both monocot and dicot plant species, including many cultivated crops. Within the species, more than 50 pathovars (i.e., pathogenic varieties, specifically causing infection to one or few host plants in most cases ) have been described [1]. The type III secretion enables to inject into the plant cell a vast arrays of proteins that, in the compatible interaction with the host, subverte the plant defence mechanisms and greatly contribute to start the infection process. Such proteins are known as effector proteins or effectors [2]. Each strain possess a unique set of effectors, also called repertoire, that may include between 20 to 33 different effectors, whereas the $P$. syringae complex as a whole shows a number of validated effector families of about 50 [3]. Each effector, in addition to being horizontally transferred, can mutate to adapt

*Corresponding author:

Email: simone.marcelletti@virgilio.it

http://dx.doi.org/10.20530/EJB 4 1-5

ISSN 2056-9912 @ 2016 to a new host plant and/or cultivar by a genetic mechanism called pathoadaptation [4].

For the relevance in causing plant disease, the establishment of the correct effector repertoire of each single strain can greatly contribute to elucidate their similarities/differences in the interaction with the host. However, the great number of effector families and the abundance of mutations and recombinations makes the reliable comparison of effector repertoires a hard task. Given the large diversity of effectors and their involvement in pathoadaptation, a correct identification requires the comparison with a large panel of genomes, but this introduces the noise associated with poor or obsolete annotation of past released genomes. As a matter of fact, the identification and assignment to families of the effectors presently requires specific manual curation and cannot be satisfactorily carried out by automated annotation procedures.

As a recent example, Templeton et al. [5] observed that for a reliable effector proteins identification both the widely used NCBI Prokariotic Genome Annotation Pipeline (PGAP) (http://www.ncbi.nlm.nih.gov/genome/annotation 
Table1. Results of comparison between the automatic annotation using NCBI database and the EffectorAnnotator software.

\begin{tabular}{llll}
\hline Orf number & \multicolumn{2}{c}{ Result software } & Result annotation \\
\hline orf00062FRU11contig41 & hopAX1.689 & FullMatch & hypothetical protein \\
orf00063FRU11contig52 & hopI1.243 & FullMatch & molecular chaperone DnaJ \\
orf00087FRU11contig53 & hopAG1.577 & FullMatch & type III effector \\
orf00083FRU11contig53 & hopAI1.609 & FullMatch & type III effector \\
orf00007FRU11contig71 & hopAH1.597 & FullMatch & type III effector \\
orf00045FRU11contig87 & hopBF1.734 & FullMatch & type III effector HopBF1 \\
orf00134FRU11contig116 & hopAA1.492 & FullMatch & type III effector \\
orf00131FRU11contig116 & hopM1.250 & FullMatch & restriction endonuclease Eco57I \\
orf00126FRU11contig116 & avrE1.65 & FullMatch & type III effector \\
orf00078FRU11contig116 & hopZ3.466 & FullMatch & avirulence protein \\
orf00073FRU11contig67 & hopI1.239 & putative & molecular chaperone DnaJ \\
orf00051FRU11contig120 & hopI1.239 & putative & MULTISPECIES: molecular chaperone DnaJ \\
orf00028FRU11contig138 & hopI1.229 & putative & molecular chaperone DnaJ \\
orf00029FRU11contig36 & hopAZ1.717 & truncated & type III effector HopAZ1 \\
orf00117FRU11contig44 & hopB1.139 & truncated & type III effector \\
orf00085FRU11contig53 & hopAH1.583 & truncated & type III effector \\
orf00079FRU11contig116 & hopAX1.689 & truncated & type III effector HrpK \\
orf00022FRU11contig44 & & & *HopAN1 protein \\
orf00017FRU11contig91 & & & *type III helper protein HopAK1 \\
orf00020FRU11contig166 & & & *MULTISPECIES: type III effector HopJ1 \\
\hline
\end{tabular}

*Not validated as true effector in the most recent release of the effector database.

prok) and the webserver RAST (http://rast.nmpdr.org) yielded inconsistent results, so that a manual search of such proteins must be performed for each comparison.

To improve the reliability of identification, assignment and further comparison of the type III secretion system effectors of $P$. syringae, we have developed and set up a program that enables fast and automated protein effectors search and comparison by using the $P$. syringae effectors databank (that can be found at www.-pseudomonassyringae.org/pst_func_gen2.htm.) The program was validated in Linux operating system (Fedora and Ubuntu) and has been already applied to a case study[6].

\section{RESULTS}

In order to verify the efficiency of effectorAnnotator, we compared the results obtained by applying this script to the annotation of Pseudomonas syringae pv. syringae CRAFRU11 (ATSU00000000) genome obtained by using the database of protein retrieved from ftp://ftp.ncbi.nlm.nih.gov/refseq/release/bacteria (last update 15/09/2015).

The annotation using NCBI database identified 13 effectors, while the effectorAnnotator found 17 effector proteins. Moreover, within the effectors found through the annotation using NCBI database, there were a Hop helper and two effectors defined by the website http://pseudomonassyringae.org/pst_func_gen2.htm as discontinued. Therefore effectorAnnotator found seven additional effectors than the annotation: four belonged to the category full match and three were referred as putative (Table 1).

The maximum number of proteomes analyzed on a PC Intel ${ }^{\circledR}$ CoreTM i7-2600 CPU @ 3.40GHz $\times 8$ with $16 \mathrm{GiB}$ of Ram is 30 . The time spent by the script was about 793m 48.899s; during this period it identifies 841 effector proteins (Figure 1).

EffectorAnnotator can be downloaded and installed from http://www.atlasplantpathogenicbacteria.it/ written in Perl and tested on Linux. 


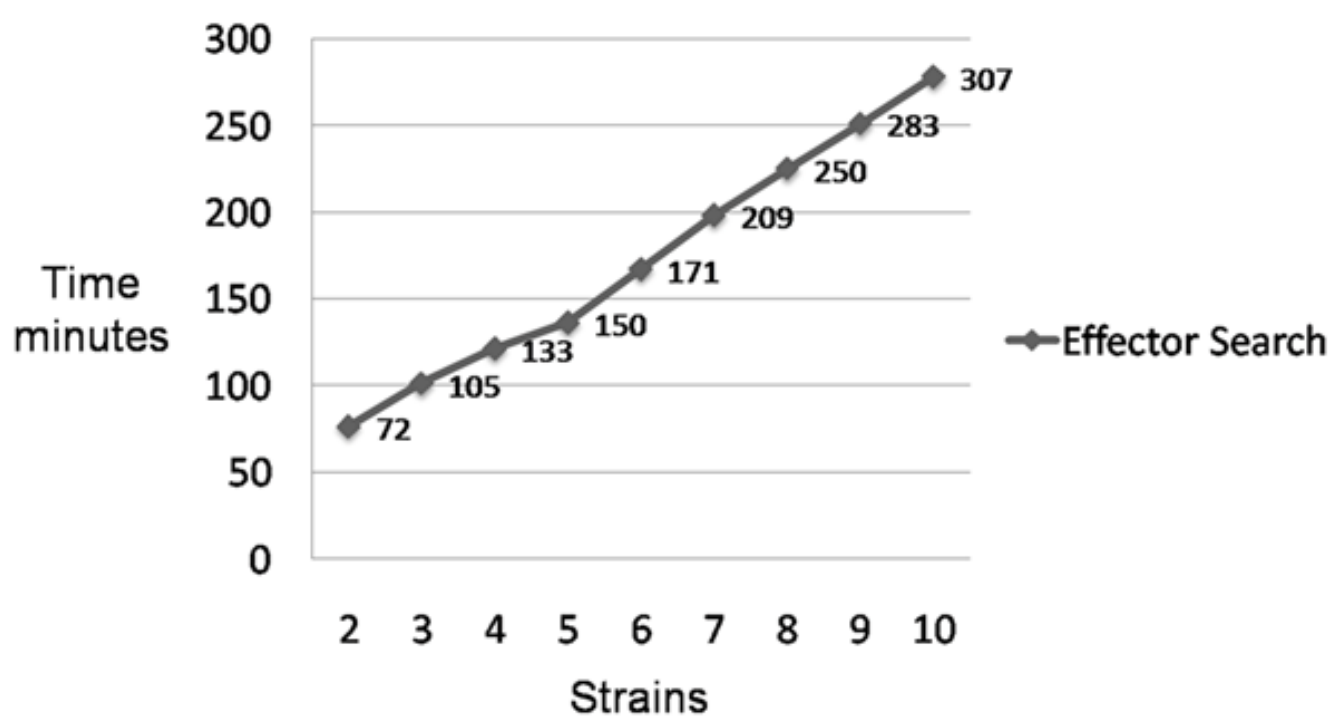

Fig. 1. Execution time ( $\mathrm{min}$ ) of EffectorAnnotator software for analyzing Pseudomonas syringae strains.

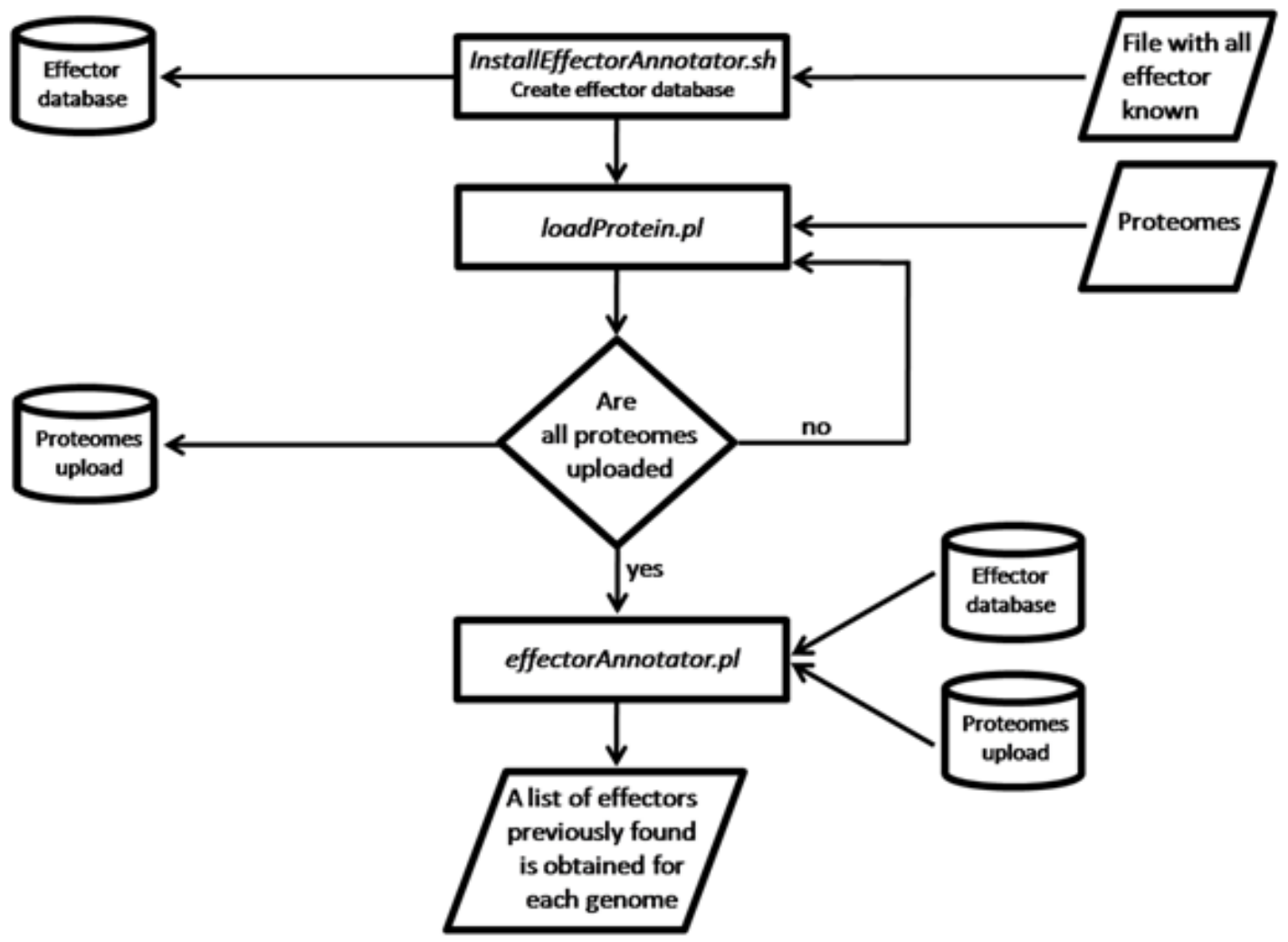

Fig. 2. Complete flow chart of EffectorAnnotator software installation and run.

\section{FUTURE DEVELOPMENTS}

In order to identify the effectors, the software exploits the bacterial genome database as an input.
Regarding to effectorAnnotator, the database includes effectors of Pseudomonas. However, by substituting or increasing the database, it would be 
also possible to use it as a tool for the identification of other effector proteins for which the automatic annotations fail. For example, it could be useful to find effectors of Xanthomonas strains by adding their protein sequences within the software.

\section{METHODS}

The software uses three external softwares. Each of them is contained in the folder "support software", BLAST v. 2.2.29 [7]; clustalW v2.0.10 [8] and PhyML v.3.1 [9].

The program is composed by five scripts: four in perl and one in bash. The script bash, installeffectorAnnotator.sh, verifies if Perl is already installed and through the script Perl, buildEffector.pl, installs the software. The script Perl loadProtein.pl uploads proteomes of bacterial genomes to be analyzed.

The script Perl effectorAnnotator.pl takes as input the effector database and the proteomes upload identifies a list of effector for each proteomes in input. Finally, Perl unistalleffectorAnnotator.pl uninstalls the software (Figure 2).

\section{Input files}

The effectorAnnotator software requires two input files: a database file containing the protein effectors (downloadable at http://pseudomonassyringae.org/pst_func_gen2.htm) and a file containing one or more (currently up to 30) proteomes of the bacterial strain(s) to be analyzed.

The effectors are classified in full match, truncated and putative, on the basis of their evalue (modifiable by the users with -e option) and alignment length: an evalue < $1 \mathrm{e}-25$ and an alignment length $>60 \%$ defines the full match effector; an evalue < $1 \mathrm{e}-25$ and an alignment length $<60 \%$ a truncated effector; an evalue > 1e-25 a putative effectors.

\section{Output files}

EffectorAnnotator generates an output (Fig1.) that includes five output files and two folders.

The histogram.jpeg file contains three different bars: FullMatchEffectors; TruncatedEffectors and PutativeEffectors.

Matrix.csv file contains the number of effectors in each strain.

Matrix2puMLST.csv file contains the presence/absence of effector in each strain.
The matrix, without the first line, can be pasted a http://pubmlst.org/cgi-

$\mathrm{bin} / \mathrm{mlstanalyse} / \mathrm{mlstanalyse} . \mathrm{pl}$ ?site=pubmlst $\&$ page $=$ treedraw\&referer=pubmlst.org to build the effector matrix tree.

The stat.txt file contains statistical information on the identified effectors.

Cytoscape.sif is a file used to create a Venn graph when used with the Venn and Euler App in Cytoscape (http://www.cytoscape.org/).

The program creates a folder containing a phylogenetic tree with effectors belonging to the same family for each bacterial strain and a folder containing phylogenetic trees comprehensive of all strain for each effector family. Files that contain phylogenetic tree can be opened with the free software figtree (http://tree.bio.ed.ac.uk/).

More extensive installation and user guides can be found in the README file.

\section{CONCLUSIONS}

EffectorAnnotator software is a rapid, simple-touse and reliable tool for the identification of effector proteins within the genome of Pseudomonas syringae strains. It allows a more accurate and reliable annotation compared to the current way of identification of such fundamental proteins involved in the pathogenicity of bacterial plant pathogens.

\section{COMPETING INTERESTS}

The authors have declared no competing interests.

\section{REFERENCE}

1. Bull, C.T., De Boer, S.H., Denny, T.P., Firrao, G., FischerLe Saux, M., Saddler, G.S., Scortichini, M., Stead, D.E. and Takikawa, Y. 2010 Comprehensive list of names of plant pathogenic bacteria, 1980-2007. Journal of Plant Pathology, 92 : 551-592.

2. Alfano JR, Collmer A. Type III secretion system effector proteins: Double Agents in Bacterial Disease and Plant Defense. Annual Review of Phytopathology. 2004 Sep;42(1):385-414. Available from: http://dx.doi.org/10.1146/annurev.phyto.42.040103. 110731.

3. Baltrus DA, Nishimura MT, Romanchuk A, Chang JH, Mukhtar MS, Cherkis K, et al. Dynamic Evolution of Pathogenicity Revealed by Sequencing and Comparative Genomics of 19 Pseudomonas syringae Isolates. Guttman DS, editor. PLoS Pathogens. 2011 Jul 

14;7(7):e1002132. Available from:
http://dx.doi.org/10.1371/journal.ppat.1002132.

4. Ma W, Dong FFT, Stavrinides J, Guttman DS. Type III Effector Diversification via Both Pathoadaptation and Horizontal Transfer in Response to a Coevolutionary Arms Race. PLoS Genet. 2006;2(12):e209. Available from:

http://dx.doi.org/10.1371/journal.pgen.0020209.

5. Templeton MD, Warren BA, Andersen MT, Rikkerink EHA, Fineran PC. Complete DNA Sequence of Pseudomonas syringae pv. actinidiae, the Causal Agent of Kiwifruit Canker Disease . Genome Announc [Internet]. American Society for Microbiology; 2015 Sep 17;3(5):e01054-15. Available from: http://dx.doi.org/10.1128/genomea.01054-15.

6. Marcelletti S, Scortichini M. Comparative Genomic Analyses of Multiple Pseudomonas Strains Infecting Corylus avellana Trees Reveal the Occurrence of Two Genetic Clusters with Both Common and Distinctive
Virulence and Fitness Traits. Semsey S, editor. PLoS ONE. 2015 Jul 6;10(7):e0131112. Available from: http://dx.doi.org/10.1371/journal.pone.0131112.

7. Altschul S. Gapped BLAST and PSI-BLAST: a new generation of protein database search programs. Nucleic Acids Research. 1997 Sep 1;25(17):3389-402. Available from: http://dx.doi.org/10.1093/nar/25.17.3389.

8. Larkin MA, Blackshields G, Brown NP, Chenna R, McGettigan PA, McWilliam $H$, et al. Clustal $W$ and Clustal X version 2.0. Bioinformatics. 2007 Sep 10;23(21):2947-8. Available from: http://dx.doi.org/10.1093/bioinformatics/btm404.

9. Guindon S, Gascuel O. A Simple, Fast, and Accurate Algorithm to Estimate Large Phylogenies by Maximum Likelihood. Systematic Biology. 2003 Oct 1;52(5):696704 Available from: http://dx.doi.org/10.1080/10635150390235520. 